\title{
Model of waterlike fluid under confinement for hydrophobic and hydrophilic particle-plate interaction potentials
}

\author{
Leandro B. Krott and Marcia C. Barbosa \\ Instituto de Física, Universidade Federal do Rio Grande do Sul, 91501-970, Porto Alegre, Rio Grande do Sul, Brazil
}

(Received 24 September 2013; revised manuscript received 20 December 2013; published 9 January 2014)

\begin{abstract}
Molecular dynamic simulations were employed to study a waterlike model confined between hydrophobic and hydrophilic plates. The phase behavior of this system is obtained for different distances between the plates and particle-plate potentials. For both hydrophobic and hydrophilic walls, there are the formation of layers. Crystallization occurs at lower temperature at the contact layer than at the middle layer. In addition, the melting temperature decreases as the plates become more hydrophobic. Similarly, the temperatures of maximum density and extremum diffusivity decrease with hydrophobicity.
\end{abstract}

DOI: 10.1103/PhysRevE.89.012110

PACS number(s): 61.20.Ja, 82.70.Dd, 83.10.Rs

\section{INTRODUCTION}

Bulk water presents a peculiar complexity on its properties. While in most materials the decrease of the temperature results in a monotonic increase of the density, the water, at ambient pressure, has a maximum in its density at $4{ }^{\circ} \mathrm{C}$ [1-3]. Furthermore, for usual liquids the response functions increase with the increase of temperature, while water exhibits an anomalous increase of compressibility [4,5] between 0.1 and $190 \mathrm{MPa}$ and, at atmospheric pressure, an increase of isobaric heat capacity upon cooling [6,7]. The anomalous behavior of water is not only related with thermodynamic functions, the diffusion coefficient for water has a maximum at $4{ }^{\circ} \mathrm{C}$ for 1.5 atm $[3,8]$, whereas for normal liquids it increases with the decrease of pressure. The anomalies have been explained in the framework of the existence of a liquid-liquid phase transition ending in a second critical point. This critical point is, however, hidden in a region of the pressure-temperature phase diagram where homogeneous nucleation takes place, and the two liquid phases do not equilibrate [9].

The difficulty of finding the liquid-liquid critical point has been circumvented by experiments performed in confined systems. In these systems, the presence of criticality in the bulk has been associated with a dynamic transition between liquids of different viscosities [10-13].

The study of water in confined geometries is, however, important not only to understand its anomalous properties, but also to learn about essential processes to the existence of life, such as enzymatic activity of proteins [14-16]. Different surfaces also induce solidification, structural jamming, and flow induced orientation [17-19]. Confined water plays an important role in many other areas such as chemistry, engineering, and geology [20-22]. For these systems, it is very important to understand the effect in the pressure-temperature phase diagram of the size of the confinement and the hydrophobicity of the wall.

Experiments with water in confined geometries employing NMR [23,24] and x-ray diffraction [25,26] show two complementary important findings. First, the pore size has important influence on the freezing and melting temperature. Next, the freezing in these systems is not uniform. Water forms layers inside the pores [27-30] that do not freeze at the same temperature [31], but the middle layers crystallize before the wall layers $[27,30]$.
Less clear than the pore size effect is the water-wall interaction effect on the melting temperature. Experimental studies show contradictory results. While Akcakayiran et al. [32] using calorimetry studies of water in pores with phosphonic, sulfonic, and carboxylic acids show that the melting temperature is not affected by the change of surface, Deschamps et al. [28] and Jelassi et al. [33] show that for water confined in hydrophobic nanopores the liquid states persist to temperatures lower than in bulk and in hydrophilic confinement. These observations are confirmed by x-ray and neutron diffraction $[33,34]$.

Simulations agree with the experiments in two points. First, the melting temperature of confined water decreases as the system becomes more restricted by decreasing the pore size or distance between plates $[35,36]$. Second, the system forms layers [37-39] where not all the confined water crystallizes $[36,40]$. The crystallized layer along the wall is in contact with a prewetting liquid layer and some systems present the formation of layers where water just crystallizes partially [40-43].

Simulations also show controversial results for the effect of hydrophobicity in the melting temperature. While results for SPC/E water show that the melting temperature for hydrophobic plates is lower than the bulk and higher than for hydrophilic walls, for the $\mathrm{mW}$ model no difference between the melting temperature due to the hydrophobicity [36] is found.

In addition to the melting line, other thermodynamic properties have been explored by simulations. In confined systems, the Temperature of Maximum Density (TMD) occurs at lower temperatures for hydrophobic confinement [40,41] and at higher temperatures for hydrophilic confinement [44] when compared with the bulk. The diffusion coefficient $D$ in the direction parallel to the plates exhibits an anomalous behavior as observed in bulk water. However, the temperatures of the the maximum and minimum of $D$ are lower than in bulk water [41]. In the direction perpendicular to the plates, no diffusion anomalous behavior is observed [45].

Atomistic and coarse-grained models [46,47] for water are a very important tool for understanding water and its properties, however, they are not appropriated for seeking for universal mechanisms that would be common for water and the other materials in which the hydrogen bonds are not present, but still they present the anomalous behavior of 


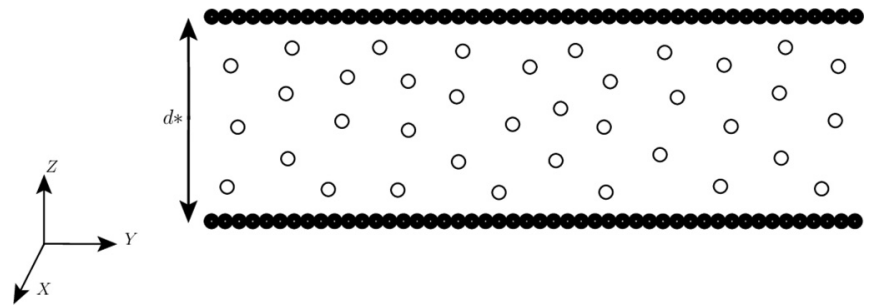

FIG. 1. Schematic depiction of the particles confined between two plates separated by a distance $d^{*}$.

water. Core-softened (CS) potentials may engender density and diffusion anomalous behavior; a number of CS potentials were proposed to model the anisotropic systems described above. They possess a repulsive core that exhibits a region of softening where the slope changes dramatically. This region can be a shoulder or a ramp [48-74]. Despite their simplicity, such models had successfully reproduced the thermodynamic, dynamic, and structural anomalous behavior present in bulk liquid water. This suggests that some of the unusual properties observed in water can be quite universal and possibly present in other systems.

In this paper, we propose that many of the properties described above can be explained qualitatively in the framework of the competition between the particle-particle interaction and the particle-plate interaction potentials. For that purpose, we study a core-softened fluid [63,64] confined between parallel plates $[75,76]$. The particle-plate interaction is then varied from a very hydrophobic to hydrophilic interaction. The pressure and temperature location of the anomalies, melting, and layering are compared with the bulk system for the different particle-plate interactions.

The paper is organized as follows: In Sec. II, we introduce the model; in Sec. III, the methods and simulation details are described; the results are given in Sec. IV; and conclusions are presented in Sec. V.

\section{MODEL}

We study systems with $N$ particles of diameter $\sigma$ confined between two fixed plates. These plates are formed by particles of diameter $\sigma$ organized in a square lattice of area $L^{2}$. The center-to-center plates distance is $d^{*}=d / \sigma$. A schematic depiction of the system is shown in Fig. 1.

The particles of the fluid interact between them through an isotropic effective potential given by

$$
\frac{U(r)}{\epsilon}=4\left[\left(\frac{\sigma}{r}\right)^{12}-\left(\frac{\sigma}{r}\right)^{6}\right]+a \exp \left[-\frac{1}{c^{2}}\left(\frac{r-r_{0}}{\sigma}\right)^{2}\right] \text {. }
$$

The first term is a standard Lennard-Jones (LJ) 12-6 potential with $\epsilon$ depth plus a Gaussian well centered on radius $r=r_{0}$ and width $c$. The parameters used are given by $a=5, r_{0} / \sigma=$ 0.7 , and $c=1$. This potential has two length scales with a repulsive shoulder at $r / \sigma \approx 1$ and a very small attractive well at $r / \sigma \approx 3.8$ (Fig. 2). This potential represents in an effective way the tetramer-tetramer interaction forming open and closed structures [77]. The pressure versus temperature

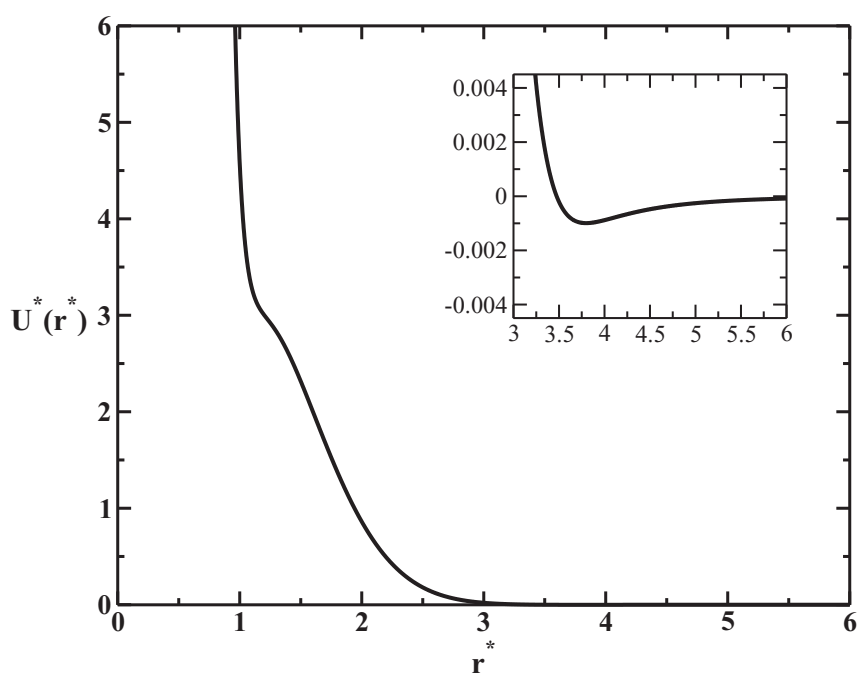

FIG. 2. Isotropic effective potential [Eq. (1)] of interaction between the waterlike particles. The energy and the distances are in dimensionless units $U^{*}=U / \epsilon$ and $r^{*}=r / \sigma$ and the parameters are $a=5, r_{0} / \sigma=0.7$, and $c=1$. The inset shows a zoom-in of the very small attractive part of the potential.

phase diagram of this system in the bulk was studied by Oliveira et al. [63,64]. Density and diffusion anomalous behavior was found for the bulk model.

In order to check the effect of hydrophobicity and confinement, five types of particle-plate interaction potentials were studied, namely, the repulsive of 24th power (R24), of 6th power (R6), Weeks-Chandler-Andersen (WCA) [78], a weak attractive (WAT), and a strong attractive (SAT). The first three of them are purely repulsive potentials, while the other two have an attractive part. Our simulations are done in reduced units, where $U^{*}=U / \epsilon$ and $r^{*}=r / \sigma$. Three purely repulsive potentials are used: R6, R24, and WCA. The equation of the more repulsive potential (R6) is

$U_{\mathrm{R} 6}^{*}=\frac{U_{\mathrm{R} 6}}{\epsilon}= \begin{cases}A_{1}(\sigma / r)^{6}+A_{2}(r / \sigma)-\varepsilon_{1}, & r \leqslant r_{c 1} \\ 0, & r>r_{c 1}\end{cases}$

where $r_{c 1}=2.0 \sigma$ and $\varepsilon_{1}=A_{1}\left(\sigma / r_{c 1}\right)^{6}+A_{2}\left(r_{c 1} / \sigma\right)$. For the R24 potential, we have

$$
U_{\mathrm{R} 24}^{*}=\frac{U_{\mathrm{R} 24}}{\epsilon}= \begin{cases}B_{1}(\sigma / r)^{24}-\varepsilon_{2}, & r \leqslant r_{c 2} \\ 0, & r>r_{c 2}\end{cases}
$$

where $r_{c 2}=1.50 \sigma$ and $\varepsilon_{2}=B_{1}\left(\sigma / r_{c 2}\right)^{24}$. The WeeksChandler-Andersen Lennard-Jones potential (WCA) is given by

$$
U_{\mathrm{WCA}}^{*}=\frac{U_{\mathrm{WCA}}}{\epsilon}= \begin{cases}U_{\mathrm{LJ}}(r)-U_{\mathrm{LJ}}\left(r_{c 3}\right), & r \leqslant r_{c 3} \\ 0, & r>r_{c 3}\end{cases}
$$

where $U_{\mathrm{LJ}}(r)$ is a standard $12-6 \mathrm{LJ}$ potential and $r_{c 3}=2^{1 / 6} \sigma$.

Two hydrophilic potentials are analyzed: one weakly hydrophilic (WAT) and another stronger (SAT). The hydrophilic 
WAT potential is given by

$$
U_{\mathrm{WAT}}^{*}=\frac{U_{\mathrm{WAT}}}{\epsilon}=\left\{\begin{array}{cc}
C_{1}\left[(\sigma / r)^{12}-(\sigma / r)^{6}\right]+C_{2}(r / \sigma)-\varepsilon_{4}, & r \leqslant r_{c 4} \\
0, & r>r_{c 4}
\end{array}\right.
$$

where $r_{c 4}=1.5 \sigma$ and $\varepsilon_{4}=C_{1}\left[\left(\sigma / r_{c 4}\right)^{12}-\left(\sigma / r_{c 4}\right)^{6}\right]+C_{2}\left(r_{c 4} / \sigma\right)$. The equation for the SAT potential is

$$
U_{\mathrm{SAT}}^{*}=\frac{U_{\mathrm{SAT}}}{\epsilon}= \begin{cases}D_{1}\left[(\sigma / r)^{12}-(\sigma / r)^{6}\right]+D_{2}(r / \sigma)-\varepsilon_{5}, & r \leqslant r_{c 5} \\ 0, & r>r_{c 5}\end{cases}
$$

where $r_{c 5}=2.0 \sigma$ and $\varepsilon_{5}=D_{1}\left[\left(\sigma / r_{c 5}\right)^{12}-\left(\sigma / r_{c 5}\right)^{6}\right]+$ $D_{2}\left(r_{c 5} / \sigma\right)$.

The parameters are illustrated in Table I.

Figure 3 illustrates the particle-plate interaction potentials. In our case, the values of the work from bringing a particle to $r^{*}=1.126$ are $-0.234,-0.061,0,0.233$, and 1.738 for the R6, R24, WCA, WAT, and SAT potentials, respectively. This confirms that the R6 potential is the most hydrophobic and the SAT is the most hydrophilic.

\section{METHODS AND SIMULATION DETAILS}

The systems are formed by $N$ particles confined in the $z$ direction by two atomically smooth plates, located each at $z=0$ and $d$. Each plate has just one layer of particles of diameter $\sigma$ organized in a square lattice of area $L^{2}$. For each phase diagram studied, the number of particles and the distance between the plates were kept fixed, so each isochore will have a different value of $L$ from $L=20 \sigma$ to $40 \sigma$. The position of each plate is fixed. To simulate infinite systems in the $x$ and $y$ directions, in order to have the thermodynamic limit, we employed periodic boundary conditions in them. Due to the empty space near each plate, the distance $d$ between them needs to be corrected to an effective distance $[41,79] d_{e}$ that can be approached by $d_{e} \approx d-\sigma$. Consequently, the effective density will be $\rho_{e}=N /\left(d_{e} L^{2}\right)$.

We use our own code in molecular dynamic simulations at the $N V T$-constant ensemble to study the problems suggested. In order to keep the temperature fixed, the Nose-Hoover $[80,81]$ thermostat with coupling parameter $Q=2$ was employed. The particle-particle interaction was done until the cutoff radius $r_{c}=3.5 \sigma$ and the potential was shifted in order to have $U=0$ at $r_{c}$.

For all the particle-plate interaction potentials, we study systems with plates separated by distances $d^{*}=d / \sigma=4.2$, 6.0, and 10.0. The properties of each case were studied for several temperatures and densities to obtain the full phase diagrams. We use $N=507$ particles for systems at $d^{*}=4.2$ and 6.0, and $N=546$ particles for systems at $d^{*}=10.0$. The initial configuration was set both on solid and liquid structures

TABLE I. Parameters of the particle-plate potentials.

\begin{tabular}{lcc}
\hline \hline Potential & Parameter values & Parameter values \\
\hline R6 & $A_{1}=4.0$ & $A_{2}=0.1875$ \\
R24 & $B_{1}=4.0$ & \\
WAT & $C_{1}=1.0$ & $C_{2}=0.289$ \\
SAT & $D_{1}=1.2$ & $D_{2}=0.0545$ \\
\hline \hline
\end{tabular}

and the equilibrium states reached after $2 \times 10^{6}$ steps, followed by a $4 \times 10^{6}$ simulation run. The time step was 0.001 in reduced units and the average of the physical quantities was obtained every 50 time steps just after the systems to be equilibrated. The use of initial conditions in the solid and liquid phases allows us to identify the final state, avoiding metastability. We used the behavior of the energy after the equilibrium states and the parallel and perpendicular pressure as a function of density to check the thermodynamic stability.

In confined systems, it is appropriated to compute the thermodynamic averages in the components parallel and perpendicular to the plates. The Helmholtz free energy is, therefore, given in terms of area $A=L_{x} L_{y}$, and distance between the plates $L_{z}$ [82]. Considering the periodic boundary conditions in the plane, the system is infinite just in the area but not in the distance between the plates. Therefore, only the parallel pressure can be regarded as a thermodynamic quantity and it might scale as the experimental pressure. Considering that, we are interested just in the quantities related to parallel direction.

The parallel pressure was calculated using the virial expression for the $x$ and $y$ directions [40,41,45,79,82,83]. The dynamics of the systems was studied by lateral diffusion coefficient $D_{\|}$, related with the mean square displacement (MSD) from the Einstein relation,

$$
D_{\|}=\lim _{\tau \rightarrow \infty} \frac{\left\langle\Delta r_{\|}(\tau)^{2}\right\rangle}{4 \tau}
$$

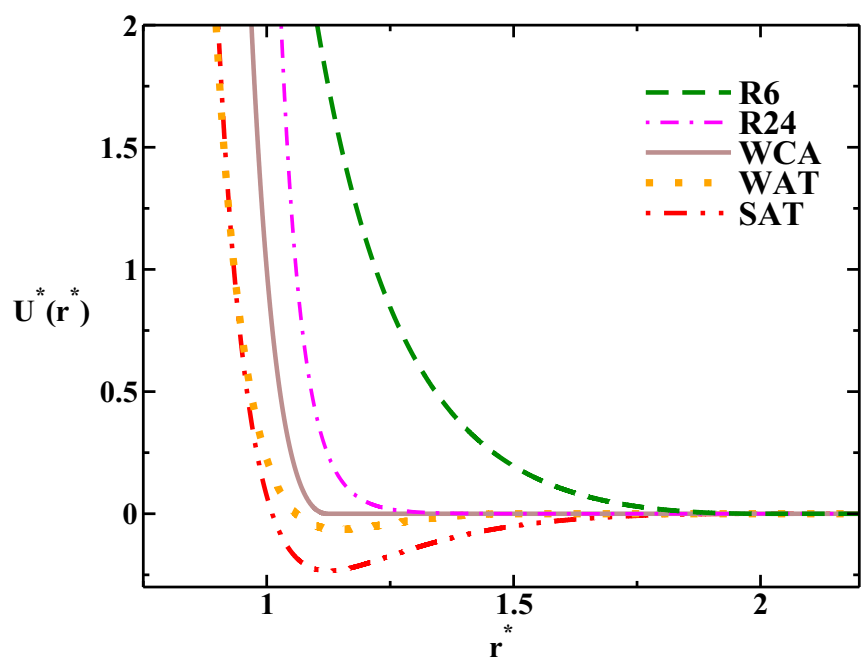

FIG. 3. (Color online) Particle-plate interaction potentials: the three purely repulsive, R6 (dashed line), R24 (dotted-dashed line), and WCA (solid line), and the attractive, WAT (dotted line), and SAT (double-dotted-dashed line). 
(a)
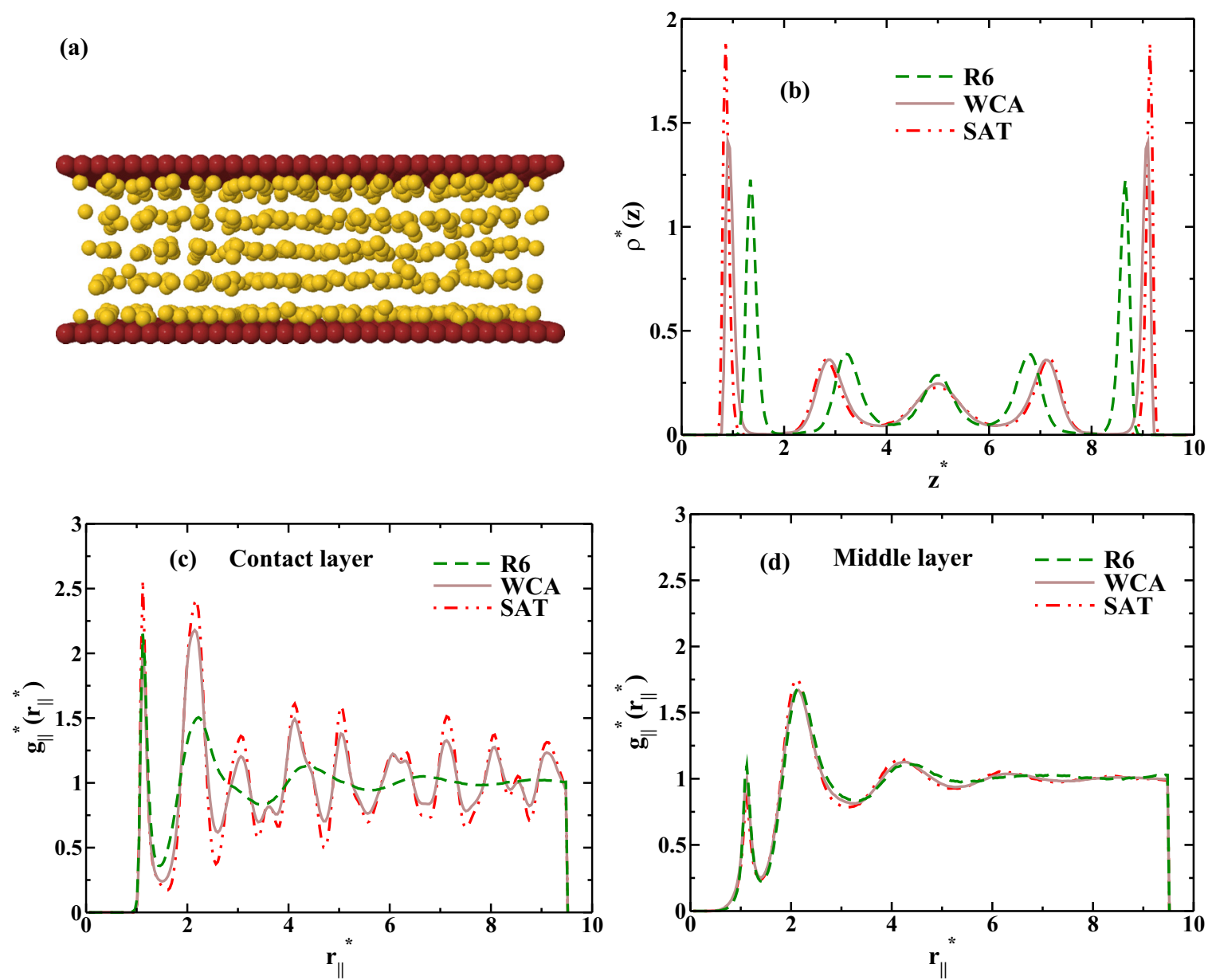

FIG. 4. (Color online) Systems with plates separated by a distance $d^{*}=10.0$ at $\rho^{*}=0.168$ and $T^{*}=0.140$. (a) Snapshot showing the five layers for the WCA case. (b) Transversal density versus $z$ for systems confined by the R6, WCA, and SAT potentials. Radial distribution function versus distance for the (c) contact and (d) middle layers. The confinements by the R24 and WAT potentials have similar results than the WCA and are not shown for simplicity.

where $r_{\|}=\left(x^{2}+y^{2}\right)^{1 / 2}$ is the distance between the particles parallel to the plates.

We also studied the structure of the systems by lateral radial distribution function $g_{\|}\left(r_{\|}\right)$. We calculate the $g_{\|}\left(r_{\|}\right)$in specific regions between the plates. A usual definition for $g_{\|}\left(r_{\|}\right)$is

$$
\begin{aligned}
& g_{\|}\left(r_{\|}\right) \\
& \quad \equiv \frac{1}{\rho^{2} V} \sum_{i \neq j} \delta\left(r-r_{i j}\right)\left[\theta\left(\left|z_{i}-z_{j}\right|\right)-\theta\left(\left|z_{i}-z_{j}\right|-\delta z\right)\right] .
\end{aligned}
$$

The $\theta(x)$ is the Heaviside function and it restricts the sum of particle pairs in the same slab of thickness $\delta z=1$. We need to compute the number of particles for each region and the normalization volume will be cylindrical. The $g_{\|}\left(r_{\|}\right)$is proportional to the probability of finding a particle at a distance $r_{\|}$from a referent particle.

All physical quantities are shown in reduced units [84] as

$$
\begin{aligned}
d^{*} & =\frac{d}{\sigma}, \quad \tau^{*}=\frac{(\epsilon / m)^{1 / 2}}{\sigma} \tau, \quad T^{*}=\frac{k_{B}}{\epsilon} T, \\
P_{\|, \perp}^{*} & =\frac{\sigma^{3}}{\epsilon} P_{\|, \perp}, \quad \rho^{*}=\sigma^{3} \rho, \quad D_{\|}^{*}=\frac{(m / \epsilon)^{1 / 2}}{\sigma} D_{\|} .
\end{aligned}
$$

\section{RESULTS}

\section{A. Structure}

First, we check the effects of decreasing the plates' distance and the hydrophobicity in the number of layers of water and its structure. For that purpose, we focus in three distances between the plates $d^{*}=10.0,6.0$, and 4.2 in which we observe the presence of five, three, and two layers, respectively, for all the particle-plate potentials.

Figure 4 illustrates the structure for the distance between the plates $d^{*}=10.0$ at $\rho^{*}=0.168$ and $T^{*}=0.140$ only for the three cases R6, WCA, and SAT for simplicity. In Fig. 4(a), the snapshot shows the structure with five layers (only the WCA for simplicity). In Fig. 4(b), the density at the $z$ direction is plotted against $z$, showing that for the attractive potential the contact layer is closer to the plates when compared with the purely repulsive particle-plate potentials. The distance between the layers is arranged to minimize the particle-particle interaction illustrated in Fig. 2, while the distance between the plate and the contact layer to minimize the particle-plate interaction. This simple geometrical arrangement is robust for all the potentials and as we shall see in the following for various plate-plate distances. Figures 4(c) and 4(d) illustrate the radial distribution functions for the 
(a)

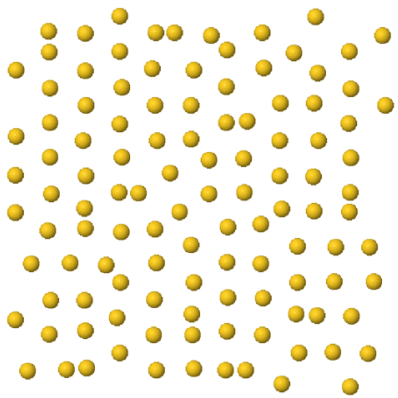

(c)

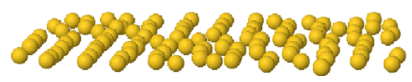

(b)

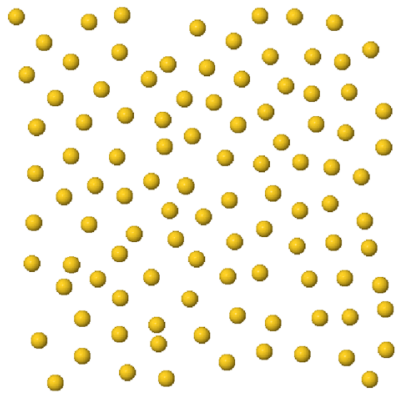

(d)

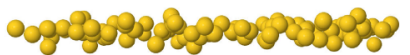

FIG. 5. (Color online) Systems confined by SAT potential with plates separated by $d^{*}=10$, for $\rho^{*}=0.125$ and $T^{*}=0.140$. Snapshots from the top of the (a) contact layer and (b) the middle layer and snapshots from the lateral of the (c) contact layer and (d) the middle layer. The lateral snapshots (c) and (d) show clearly the difference in the structure of each layer.

contact and middle layers, respectively. While the contact layers show the presence of an amorphouslike structure, the middle layers are liquid. The only case in which both layers are liquids is the repulsive case R6. This result is in agreement with SPC/E simulation of Gallo et al. [85].

Figure 5 shows the snapshots of a system confined by SAT potential with plates separated by $d^{*}=10$, for $\rho^{*}=0.125$ and $T^{*}=0.140$. In Figs. 5(a) and 5(b), we see the top of the contact and middle layers, respectively, while in Figs. 5(c) and 5(d) a lateral view is shown for the same layers. The contact layer exhibits some order while the central layer is liquid. Even with the presence of some order, the contact layer is more likely in an amorphous phase.

Figure 6 illustrates the system for plates separated by a distance $d^{*}=6.0$ at $\rho^{*}=0.150$ and $T^{*}=0.140$. In Fig. 6(a), the snapshot shows the structure with three layers (only the WCA for simplicity): two contact layers and one middle layer. In Fig. 6(b), the density versus $z$ indicates that as the plates become more attractive, particles are pushed toward them. Figures 6(c) and 6(d) show that for $d^{*}=6.0$, the contact layer presents an amorphouslike structure, while the middle layer is fluid. This observation is true for all the particle-plate potentials with the exception of the R6, which is fluid at the contact and middle layers.

For the distance $d^{*}=4.2$ and $\rho^{*}=0.165$, two temperatures were analyzed: $T^{*}=0.140$ and 0.250 . Figure 7 (a) shows

(a)
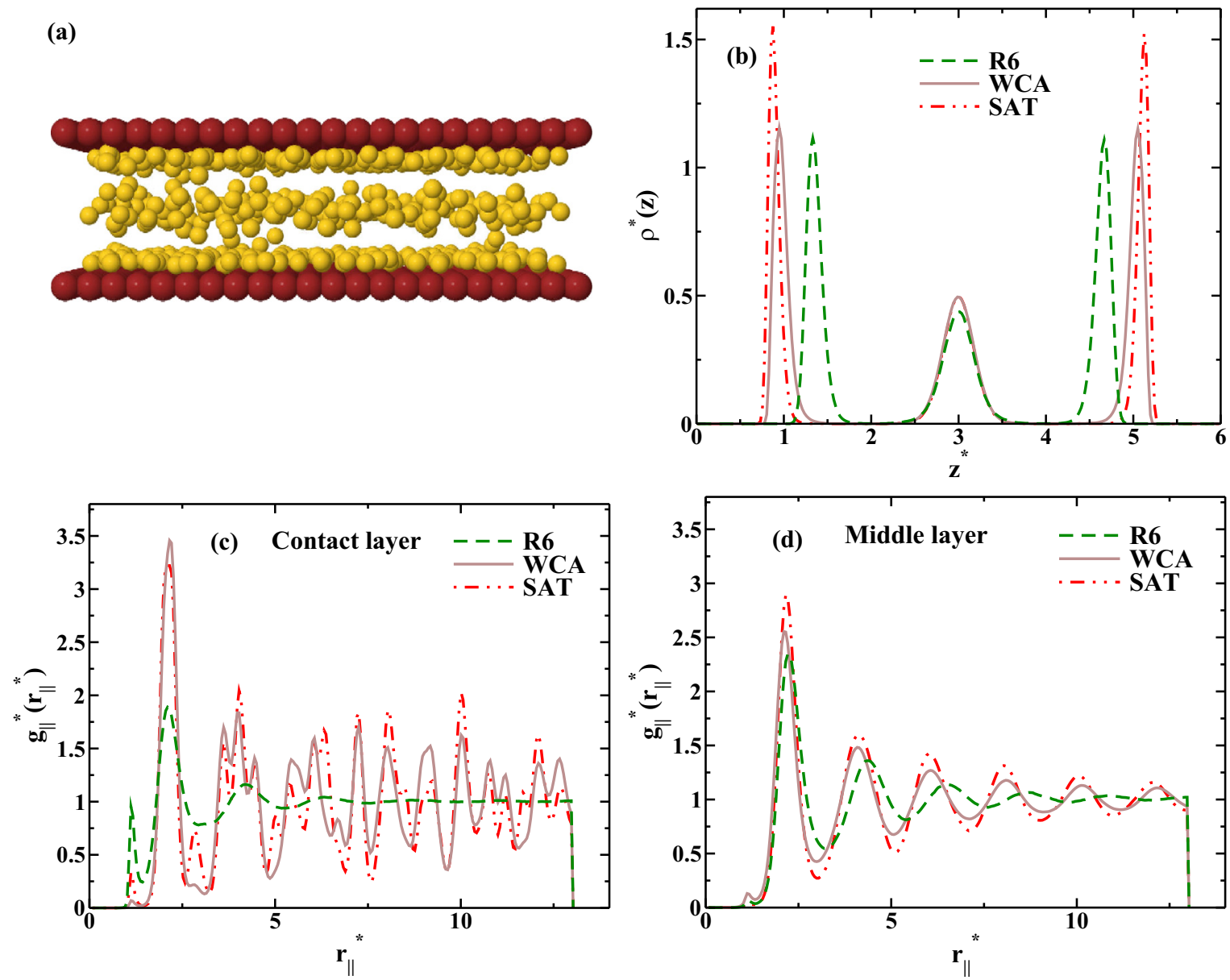

FIG. 6. (Color online) Systems with plates separated by a distance $d^{*}=6.0$ at $\rho^{*}=0.150$ and $T^{*}=0.140$. (a) Snapshot showing the three layers for the WCA case. (b) Transversal density versus $z$ for systems confined by the R6, WCA, and SAT potentials. Radial distribution function versus distance for the (c) contact and (d) middle layers. The confinements by the R24 and WAT potentials have similar results than the WCA and are not shown for simplicity. 
(a)
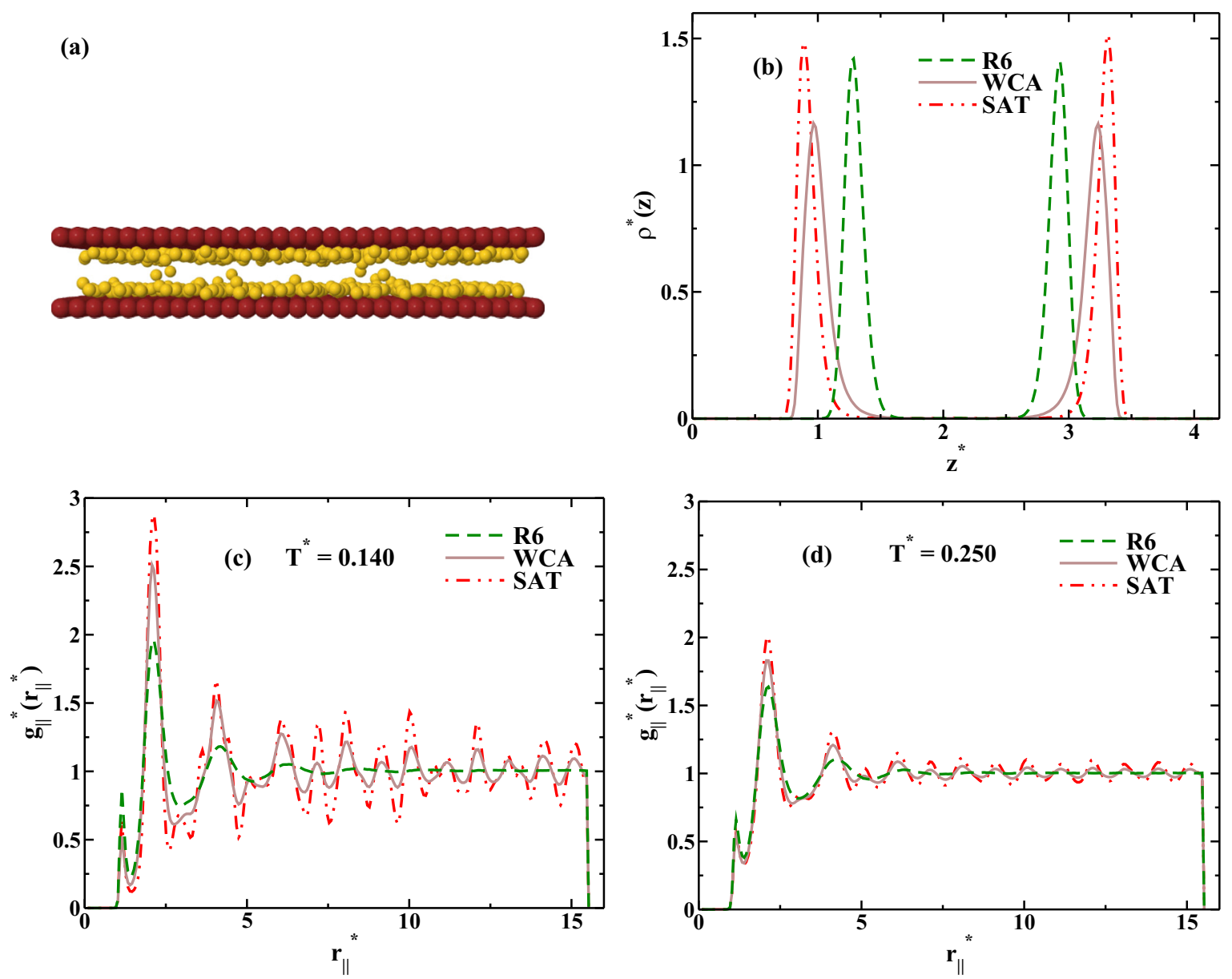

FIG. 7. (Color online) Systems with plates separated by a distance $d^{*}=4.2$ at $\rho^{*}=0.165$. (a) Snapshot showing the two layers for the WCA case at $T^{*}=0.140$. (b) Transversal density versus $z$ for systems confined by the R6, WCA, and SAT potentials. Radial distribution function versus distance for one contact layer for (c) $T^{*}=0.140$ and for (d) $T^{*}=0.250$. The confinements by the R24 and WAT potentials have similar results than the WCA and are not shown for simplicity.

a snapshot of the system (only for the WCA for simplicity) indicating the presence of two layers. Figure 7(b) shows the density at the $z$ direction. Similarly to the $d^{*}=10$ and 6.0 cases, the main effect of hydrophobicity is to have the two layers closer to the wall than in the case of the hydrophilic wall. Figures 7(c) and 7(d) show the radial distribution function for
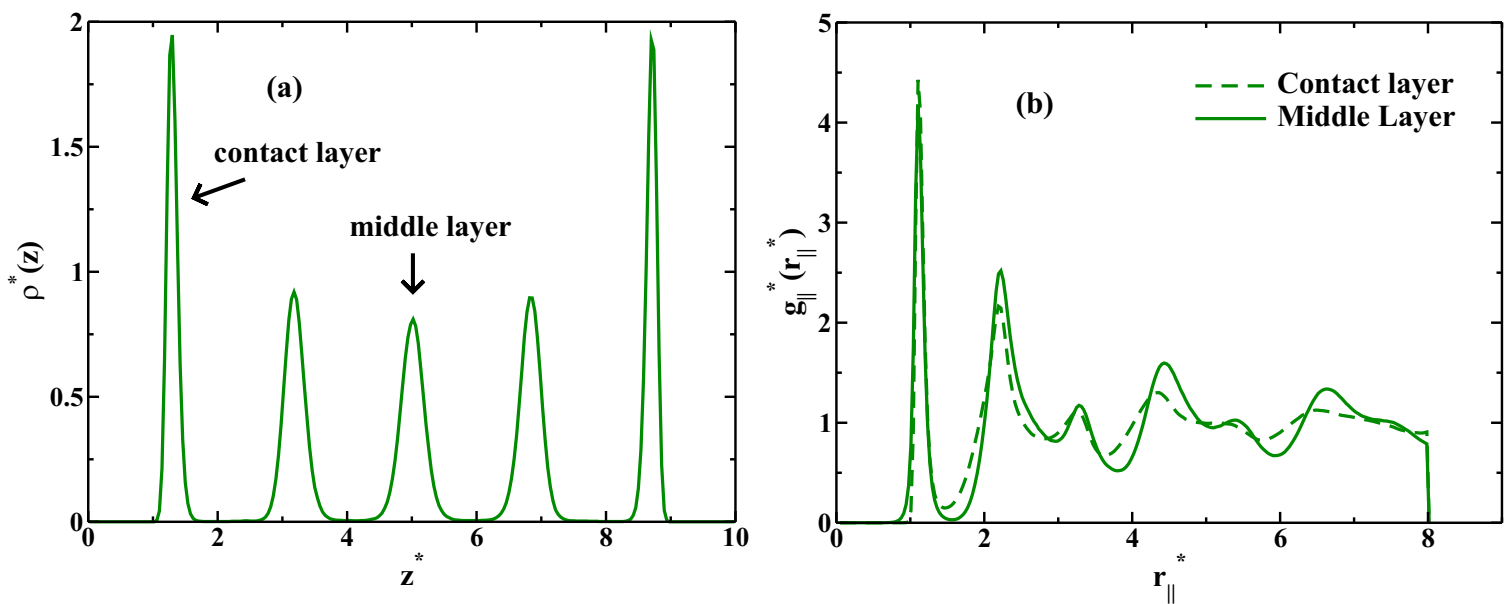

FIG. 8. (Color online) Transversal density versus $z$ in (a) and $g_{\|}\left(r_{\|}\right)$in (b) for $d^{*}=10.0, \rho^{*}=0.209$, and $T^{*}=0.140$. The middle layer is more structured than the contact layer, which is in agreement with some experimental results for hydrophobic confinement. 

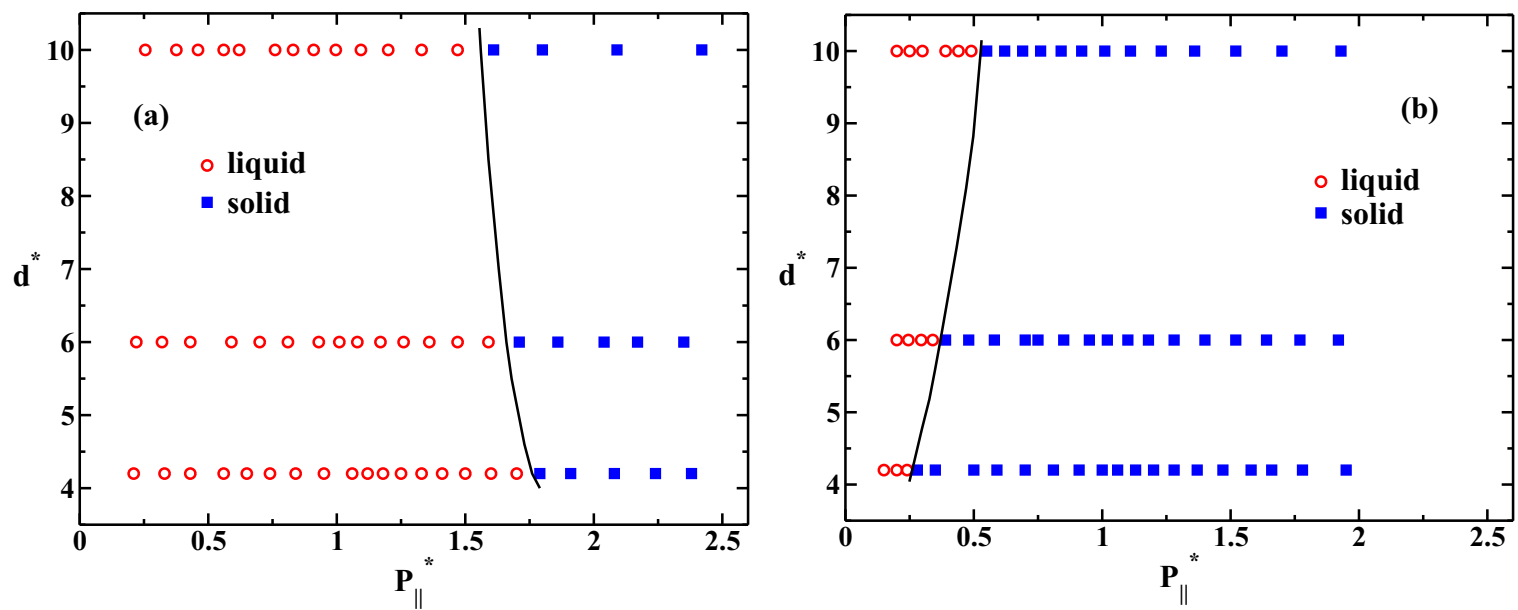

FIG. 9. (Color online) Distance $d^{*}$ between the plates as a function of parallel pressure of the system for (a) R6 and (b) SAT confinements at $T^{*}=0.140$. The open circles indicate liquid-state points while filled squares indicate solid-state points. The black lines are an approximate boundary between the liquid and solid states.

two temperatures $T^{*}=0.140$ and $T^{*}=0.250$, respectively. While $T^{*}=0.140$ shows an amorphouslike structure for the WCA and SAT potentials, $T^{*}=0.250$ is liquid, indicating that the system melts at an intermediate temperature. The R6 potentials exhibit a liquidlike behavior for both temperatures.

In all the cases shown above, the purely repulsive potential R6 has no crystalline layer. This suggests that the crystallization in this case occurs at higher pressures. In order to check that, the case at $d^{*}=10.0$ is analyzed for $\rho^{*}=0.209$ in comparison with $\rho^{*}=0.168$, shown in Fig. 4. In Fig. 8(a), the transversal density versus $z$ is shown for a system with plates separated by $d^{*}=10.0$ at $\rho^{*}=0.209$ and $T^{*}=0.140$, showing the five layers formed, and in Fig. 8(b), we have the $g_{\|}\left(r_{\|}\right)$of the contact and the middle layers. Both layers represent amorphous states. In addition, it is possible to see that the middle layer is smoothly more structured than the contact layer. This result is consistent with experiments [27,30] that show that the middle layer crystallizes at higher temperatures when compared with the contact layer. This result is obtained just for the R6 potential, which leads us again to the conclusion that this potential is the best to reproduce the structure related in some experiments for the hydrophobicity.

Our results, comparing the different potentials and plates distances, indicate that the hydrophobicity has little effect in the number of layers that is defined by the distance $d^{*}$ between the plates. The crystallization of the contact layer, however, seems to be dependent both of the particle-plate interaction and of the distance between the plates. In order to explore in detail the process of crystallization of the contact layer, we analyze the phase behavior of the confined systems for the R6 and SAT potentials. Figure 9 shows the phase behavior of the systems confined by the (a) R6 and (b) SAT potentials at $T^{*}=0.140$. The open circles indicate liquid-state points while filled squares indicate solid-state points. An approximate boundary between the liquid and solid states is indicated by the black lines in the figures. For this specific temperature, our results suggest that the melting pressure decreases with $d^{*}$ for the hydrophobic potentials and increases with $d^{*}$ for the hydrophilic potentials. Molecular systems confined by strongly attractive surfaces present a
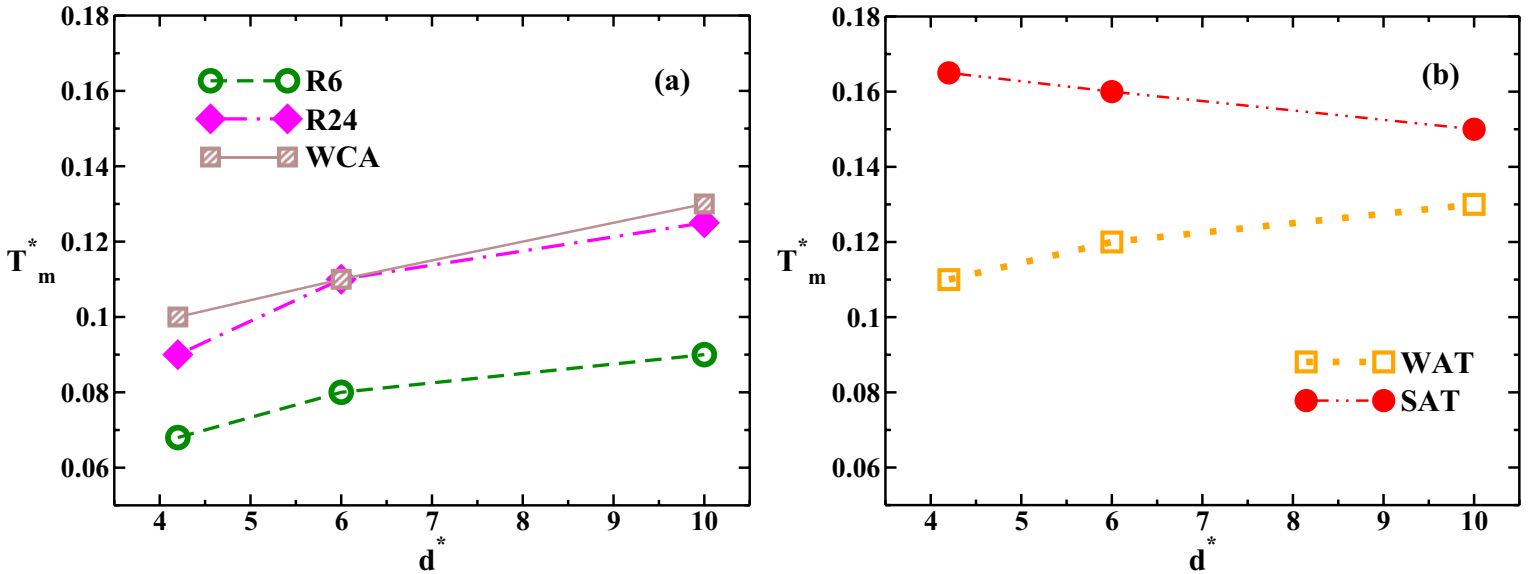

FIG. 10. (Color online) Melting temperature as a function of separation $d^{*}$ between the plates for (a) repulsive (R6, R24, and WCA) and (b) attractive (WAT and SAT) potentials. Systems at $\rho^{*}=0.176$. 

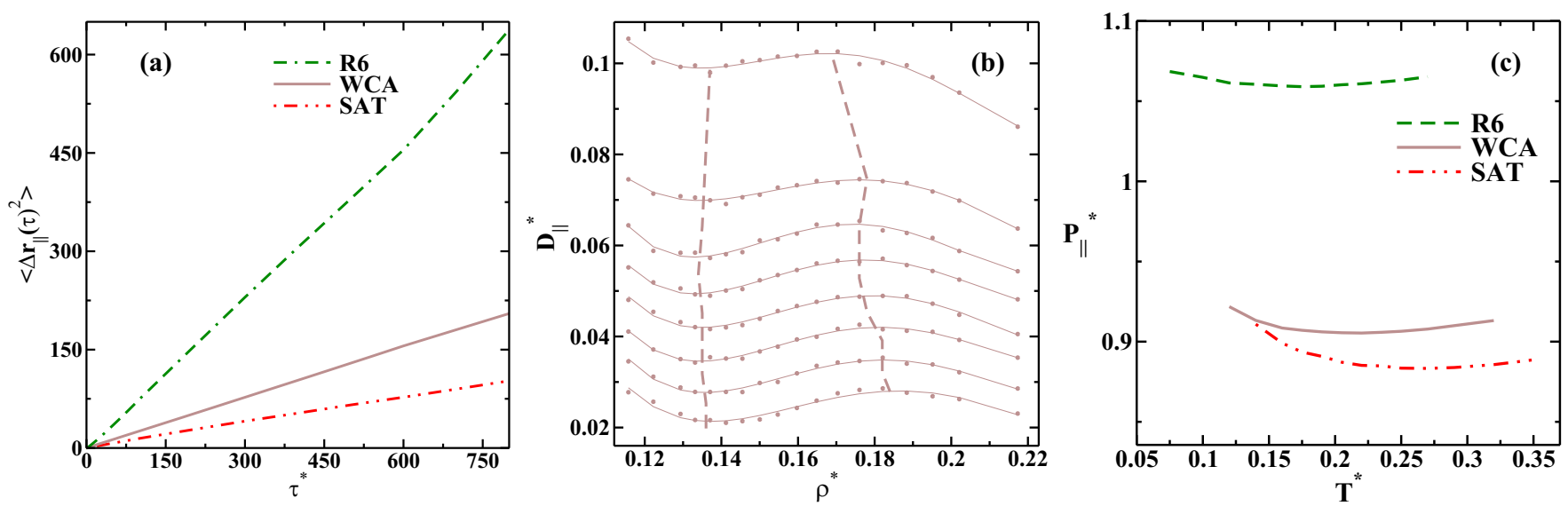

FIG. 11. (Color online) Systems for $d^{*}=4.2, \rho^{*}=0.165$, and $T^{*}=0.250$. (a) Mean square displacement versus time for R6, WCA, and SAT potentials. (b) Diffusion coefficient versus density for the WCA potential at fixed temperatures $T^{*}=0.175,0.190,0.205,0.220,0.235$, $0.250,0.270$, and 0.320 from the bottom to the top. (c) Isochore $\rho^{*}=0.165$ at the pressure-temperature phase diagram for R6, WCA, and SAT potentials. The R24 and WAT potentials are intermediate cases and are not shown for simplicity.
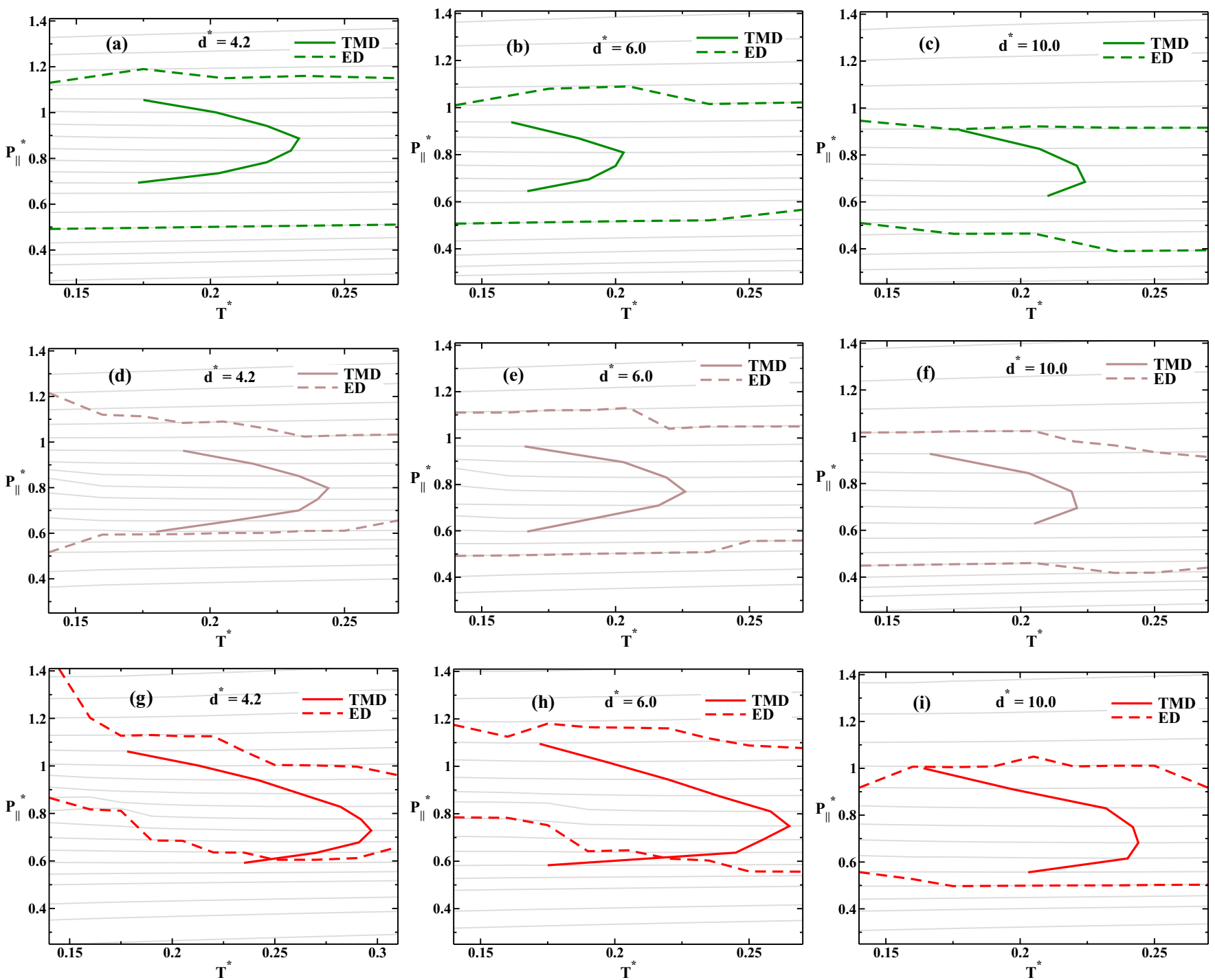

FIG. 12. (Color online) Parallel pressure versus temperature phase diagrams for R6 [(a)-(c)], WCA [(d)-(f)], and SAT [(g)-(i)] potentials. For all the plots, the solid line is the TMD line and the dashed line is the extremum diffusion coefficient line. The ranges of densities are $0.089 \leqslant \rho^{*} \leqslant 0.182$ for systems at $d^{*}=4.2,0.087 \leqslant \rho^{*} \leqslant 0.176$ for $d^{*}=6.0$, and $0.083 \leqslant \rho^{*} \leqslant 0.168$ for $d^{*}=10.0$. 

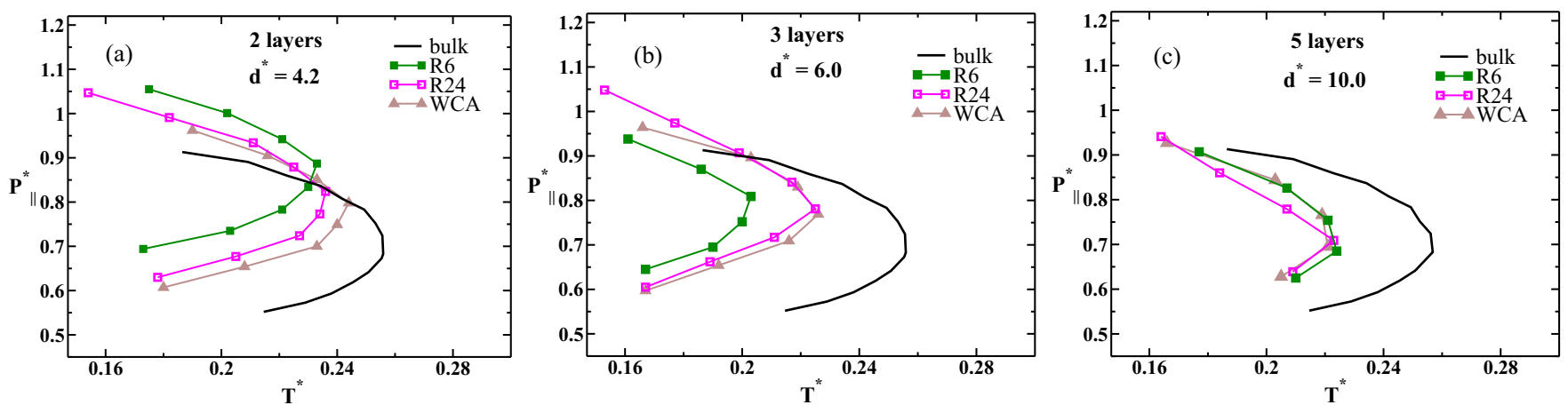

FIG. 13. (Color online) Pressure versus temperature phase diagram illustrating the TMD line for hydrophobic confinement, R6, R24, and WCA potentials, for (a) $d^{*}=4.2$, (b) $d^{*}=6.0$, and (c) $d^{*}=10.0$.

similar behavior in relation to melting temperature, aside from the order-promoting effect to be thickness dependent $[17,86,87]$. These results also are consistent with the liquid-gas observations in the SPC/E confined model [35].

Figure 10 shows the melting temperature of the systems at $\rho^{*}=0.176$ for (a) repulsive potentials (R6, R24, and WCA) and for (b) attractive potentials (WAT and SAT). For temperatures $T^{*}>T_{m}^{*}$, all the systems are in liquid state, while for $T^{*}<T_{m}^{*}$ a crystallization occurs at least for the contact layers. The SAT potential crystallizes more easily than the other cases and has a peculiar behavior with the distance $d^{*}$ between the plates. The crystallization for the SAT potentials occurs more easily as the degree of confinement increases (decreasing of $d^{*}$ ), while other cases show the opposite behavior. This result indicates that hydrophobic walls have the effect of disordering the system since the wall-particle repulsion does not promote the fluid particles to follow the wall arrangement. For attractive wall-particle interactions, the wall orders the particles of the fluid according to its distribution. Our results are in agreement with simulations [20,22,36] and experiments [27,28] for water confined in hydrophobic and hydrophilic nanopores and for a Lennard-Jones methane confined by cylindrical pores [88].

It is interesting to compare the results for this confined model with the bulk results. For the range of densities and temperatures studied in this work, the melting temperature presented different behaviors in relation to bulk. For example, at $\rho^{*}=0.176$, the melting temperature of the bulk is about $T_{m}^{*}=0.100[63,64,89]$. For the R6 confinement, $T_{m}^{*}<0.090$ for the three separations of the plates studied, while that for the SAT potential, $T_{m}^{*}>0.150$.

\section{B. Diffusion and density anomalous behavior}

In this section, we analyze the effect of hydrophobicity and changing the plates' distances in the location in the pressure-temperature phase diagram of the diffusion and density anomalies. Figure 11(a) shows a comparison between the mean square displacement parallel to the plates at $d^{*}=4.2, \rho^{*}=0.165$, and $T^{*}=0.250$. The plot shows that the mobility is higher for hydrophobic than hydrophilic particle-plate interactions. This result is consistent with the layer density illustrated in Fig. 7(b) that shows that attractive particle-plate interactions leave more space for the layers. In Fig. 11(b), the diffusion coefficient is shown as a function of the density for $d^{*}=4.2$ and WCA confining potential, illustrating the presence of a region where diffusion increases with the increase of the density that is defined as the diffusion anomalous region (region between the dashed lines). This anomalous behavior is also present for the other distances $d=6.0$ and 10.0 and other particle-plate potentials.

Next, we test our system for the presence of the temperature of maximum density (TMD). The TMD lines can be found computing $\left(\partial P_{\|} / \partial T\right)_{\rho}=0$, corresponding to minimum of the isochores. A comparison between the same isochore $\left(\rho^{*}=0.165\right)$ for each potential is given in Fig. 11(c) for $d^{*}=4.2$. The temperature of maximum density decreases and its pressure increases as the system becomes more hydrophobic. The pressure increase can be understood in
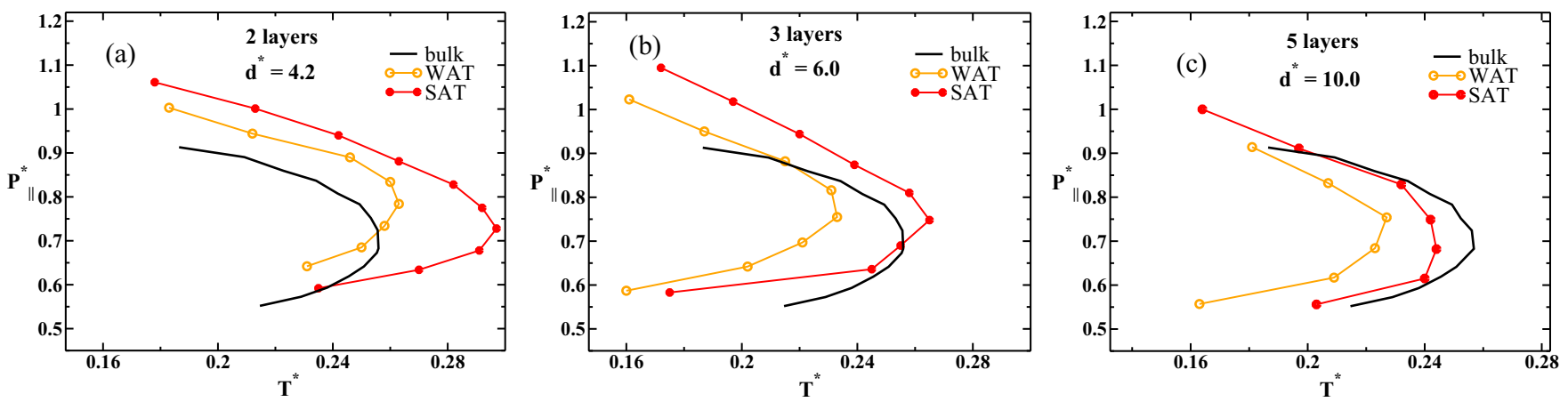

FIG. 14. (Color online) Pressure versus temperature phase diagram illustrating the TMD line for hydrophilic confinement, WAT and SAT potentials, for (a) $d^{*}=4.2$, (b) $d^{*}=6.0$, and (c) $d^{*}=10.0$. 
terms of the decrease of effective volume for hydrophobic plates as shown in Fig. 7(b) with corresponding increase of pressure. The decrease of the TMD with hydrophobicity can be understood as follows. In our effective model, the two length scales represent the bond and nonbonding cluster of molecules. As temperature increases, the number of clusters with "nonbonding" molecules grows while the number of clusters with "bonding" molecules decreases. The TMD is the temperature in which the two distributions become equivalent. In the confined system, the wall repulsion favors the nonbonding length scale and the TMD happens at lower values. Figure 12 shows the parallel pressure versus temperature phase diagram for R6 [Figs. 12(a)-12(c)], WCA [Figs. 12(d)-12(f)], and SAT [Figs. 12(g)-12(i)] potentials. The dashed lines comprise the diffusion anomaly and the solid lines indicate the density anomaly for each case. For all the cases studied, the hierarchy of the anomalies is observed.

Confirming the scenario we describe above, Fig. 13 illustrates the TMD lines for the hydrophobic particle-plate interaction potentials for (a) $d^{*}=4.2$, (b) $d^{*}=6.0$, and (c) $d^{*}=10.0$. The TMD lines are shifted to lower temperatures in relation to bulk system as the distance between the plates is decreased. This result is consistent with atomistic models [40,41].

Figure 14 shows the TMD lines for the hydrophilic particleplate interaction potentials for different plate separations. For these cases, the TMD moves to higher temperatures when compared to the bulk values as the distance between the plates is decreased. This result is consistent with atomistic models [44].

\section{CONCLUSIONS}

In this paper, we have explored the effect of the confinement in the thermodynamic, dynamic, and structural properties of a core-softened potential designed to reproduce the anomalies present in water. We have shown that both hydrophobic and hydrophilic walls change the melting, the TMD, and the extrema diffusivity temperatures. While melting is suppressed by hydrophobic walls, crystallization happens for hydrophilic confinement at higher temperatures if the walls would be attractive enough.

Our results suggest that layering, crystallization, and thermodynamic and dynamic anomalies are governed by the competition between the two length scales that characterize our model and the particle-plate interaction length. These results are consistent with atomistic models [35,36,41,43], however, due to the simplicity of the simulation, we were able to explore a large variety of potentials to confirm our assumption that a simple competition between scales not only is able to reproduce the water anomalies, but is also able to capture the confinement phase diagram.

\section{ACKNOWLEDGMENTS}

We thank for financial support the Brazilian science agencies CNPq and Capes. This work is partially supported by CNPq, INCT-FCx. We also thank the CEFIC-Centro de Física Computacional of Physics Institute at UFRGS for the computer clusters.
[1] R. Waler, Essays of Natural Experiments (Johnson Reprint, New York, 1964).

[2] G. S. Kell, J. Chem. Eng. Data 20, 97 (1975).

[3] C. A. Angell, E. D. Finch, L. A. Woolf, and P. Bach, J. Chem. Phys. 65, 3063 (1976).

[4] R. J. Speedy and C. A. Angell, J. Chem. Phys. 65, 851 (1976).

[5] H. Kanno and C. A. Angell, J. Chem. Phys. 70, 4008 (1979).

[6] C. A. Angell, M. Oguni, and W. J. Sichina, J. Chem. Phys. 86, 998 (1982).

[7] E. Tombari, C. Ferrari, and G. Salvetti, Chem. Phys. Lett. 300, 749 (1999).

[8] F. X. Prielmeier, E. W. Lang, R. J. Speedy, and H.-D. Lüdemann, Phys. Rev. Lett. 59, 1128 (1987).

[9] P. H. Poole, F. Sciortino, U. Essmann, and H. E. Stanley, Nature (London) 360, 324 (1992).

[10] L. Liu, S.-H. Chen, A. Faraone, C.-W. Yen, and C.-Y. Mou, Phys. Rev. Lett. 95, 117802 (2005).

[11] X.-Q. Chu, K.-H. Liu, M. S. Tyagi, C.-Y. Mou, and S.-H. Chen, Phys. Rev. E 82, 020501 (2010).

[12] S.-H. Chen, F. Mallamace, C.-Y. Mou, M. Broccio, C. Corsaro, A. Faraone, and L. Liu, Proc. Natl. Acad. Sci. U. S. A. 103, 12974 (2006).

[13] F. Mallamace, C. Corsaro, M. Broccio, C. Branca., N. GonzálezSegredo, J. Spooren, S.-H. Chen, and H. E. Stanley, Proc. Natl. Acad. Sci. U. S. A. 105, 12725 (2008).

[14] S.-H. Chen, L. Liu, E. Fratini, P. Baglioni, A. Faraone, and E. Mamontov, Proc. Natl. Acad. Sci. U. S. A. 103, 9012 (2006).
[15] G. Franzese, K. Stokely, X-q. Chu, P. Kumar, M. G. Mazza, S.-H. Chen, and H. E. Stanley, J. Phys.: Condens. Matter 20, 494210 (2008).

[16] A. Kuffel and J. Zielkiewicz, J. Phys. Chem. B 116, 12113 (2012).

[17] S. T. Cui, P. T. Cummings, and H. D. Cochran, J. Chem. Phys. 114, 7189 (2001).

[18] A. Jabbarzadeh, P. Harrowell, and R. I. Tanner, J. Chem. Phys. 125, 034703 (2006).

[19] A. Jabbarzadeh and R. I. Tanner, Tribol. Int. 44, 711 (2011).

[20] M. Rullich, V. C. Weiss and T.s. Frauenheim, Modell. Simul. Mater. Sci. Eng. 21, 055027 (2013).

[21] C. D. Lorenz, M. Chandross, J. M. D. Lane, and G. S. Grest, Modell. Simul. Mater. Sci. Eng. 18, 034005 (2010).

[22] L. Ramin and A. Jabbarzadeh, Langmuir 29, 13367 (2013).

[23] E. W. Hansen, M. Stöker, and R. Schmidt, J. Chem. Phys. 100, 2195 (1996).

[24] K. Overloop and L. Van Gerven, J. Magn. Res. 101, 179 (1992).

[25] K. Morishige and K. Kawano, J. Chem. Phys. 110, 4867 (1999).

[26] K. Morishige and H. Iwasaki, Langmuir 19, 2808 (2003).

[27] M. Erko, N. Cade, A. G. Michette, G. H. Findenegg, and O. Paris, Phys. Rev. B 84, 104205 (2011).

[28] J. Deschamps, F. Audonnet, N. Brodie-Linder, M. Schoeffel, and C. Alba-Simionesco, Phys. Chem. Chem. Phys. 12, 1440 (2010).

[29] S. Jähnert, F. V. Chávez, G. E. Schaumann, A. Schreiber, M. Schönhoff, and G. H. Findenegg, Phys. Chem. Chem. Phys. 10, 6039 (2008). 
[30] K. Morishige and K. Nobuoka, J. Chem. Phys. 107, 6965 (1997)

[31] M.-C. Bellissent-Funel, J. Lal, and L. Bosio, J. Chem. Phys. 98, 4246 (1993).

[32] D. Akcakayiran, D. Mauder, C. Hess, T. K. Sievers, D. G. Kurth, I. Shenderovich, H. H. Limbach, and G. H. Findenegg, J. Phys. Chem. B 112, 14637 (2008).

[33] J. Jelassi, T. Grosz, I. Bako, M.-C. Belissent-Funel, J. C. Dore, H. L. Castricum, and R. Sridi-Dorbez, J. Chem. Phys. 134, 064509 (2011).

[34] M. Sliwinska-Bartkowiak, M. Jazdzewska, L. L. Huang, and K. E. Gubbins, Phys. Chem. Chem. Phys. 10, 4909 (2008).

[35] N. Giovambattista, P. J. Rossky, and P. G. Debenedetti, J. Phys. Chem. B 113, 13723 (2009).

[36] E. B. Moore, J. T. Allen, and V. Molinero, J. Phys. Chem. C 116, 7507 (2012).

[37] R. Zangi and A. E. Mark, J. Chem. Phys. 119, 1694 (2003).

[38] S. Han, M. Y. Choi, P. Kumar, and H. E. Stanley, Nat. Phys. 6 , 685 (2010)

[39] J. R. Bordin, A. B. de Oliveira, A. Diehl, and M. C. Barbosa, J. Chem. Phys. 137, 084504 (2012).

[40] N. Giovambattista, P. J. Rossky, and P. G. Debenedetti, Phys Rev. Lett. 102, 050603 (2009).

[41] P. Kumar, S. V. Buldyrev, F. W. Starr, N. Giovambattista, and H. E. Stanley, Phys. Rev. E 72, 051503 (2005).

[42] P. Gallo, M. Rovere, and E. Spohr, J. Chem. Phys. 113, 11324 (2000).

[43] E. G. Solveyra, E. de la Llave, D. A. Scherlis, and V. Molinero, J. Phys. Chem. B 115, 14196 (2011).

[44] S. R.-V. Castrillon, N. Giovambattista, I. A. Aksay, and P. G. Debenedetti, J. Chem. Phys. B 113, 1438 (2009).

[45] S. Han, P. Kumar, and H. E. Stanley, Phys. Rev. E 77, 030201 (2008).

[46] F. Santos and G. Franzese, J. Phys. Chem. B 115, 14311 (2011).

[47] E. G. Strekalova, M. G. Mazza, H. E. Stanley, and G. Franzese, J. Phys.: Condens. Matter 24, 064111 (2012).

[48] P. C. Hemmer and G. Stell, Phys. Rev. Lett. 24, 1284 (1970).

[49] A. Scala, M. R. Sadr-Lahijany, N. Giovambattista, S. V. Buldyrev, and H. E. Stanley, J. Stat. Phys. 100, 97 (2000).

[50] S. V. Buldyrev, G. Franzese, N. Giovambattista, G. Malescio, M. R. Sadr-Lahijany, A. Scala, A. Skibinsky, and H. E. Stanley, Phys. A (Amsterdam) 304, 23 (2002).

[51] C. Buzano and M. Pretti, J. Chem. Phys. 119, 3791 (2003).

[52] A. Skibinsky, S. V. Buldyrev, G. Franzese, G. Malescio, and H. E. Stanley, Phys. Rev. E 69, 061206 (2004).

[53] G. Franzese, G. Malescio, A. Skibinsky, S. V. Buldyrev, and H. E. Stanley, Phys. Rev. E 66, 051206 (2002).

[54] A. L. Balladares and M. C. Barbosa, J. Phys.: Condens. Matter 16, 8811 (2004).

[55] A. B. de Oliveira and M. C. Barbosa, J. Phys.: Condens. Matter 17, 399 (2005).

[56] V. B. Henriques and M. C. Barbosa, Phys. Rev. E 71, 031504 (2005).

[57] V. B. Henriques, N. Guissoni, M. A. Barbosa, M. Thielo, and M. C. Barbosa, Mol. Phys. 103, 3001 (2005).

[58] E. A. Jagla, Phys. Rev. E 58, 1478 (1998).

[59] N. B. Wilding and J. E. Magee, Phys. Rev. E 66, 031509 (2002).
[60] S. Maruyama, K. Wakabayashi, and M. A. Oguni, AIP Conf. Proc. 708, 675 (2004).

[61] R. Kurita and H. Tanaka, Science 206, 845 (2004).

[62] L. Xu, P. Kumar, S. V. Buldyrev, S.-H. Chen, P. Poole, F. Sciortino, and H. E. Stanley, Proc. Natl. Acad. Sci. U. S. A. 102, 16558 (2005).

[63] A. B. de Oliveira, P. A. Netz, T. Colla, and M. C. Barbosa, J. Chem. Phys. 124, 084505 (2006)

[64] A. B. de Oliveira, P. A. Netz, T. Colla, and M. C. Barbosa, J. Chem. Phys. 125, 124503 (2006).

[65] A. B. de Oliveira, M. C. Barbosa, and P. A. Netz, Phys. A (Amsterdam) 386, 744 (2007).

[66] A. B. de Oliveira, P. A. Netz, and M. C. Barbosa, Eur. Phys. J. B 64, 481 (2008).

[67] A. B. de Oliveira, G. Franzese, P. A. Netz, and M. C. Barbosa, J. Chem. Phys. 128, 064901 (2008).

[68] A. B. de Oliveira, P. A. Netz, and M. C. Barbosa, Europhys. Lett. 85, 36001 (2009).

[69] N. V. Gribova, Y. D. Fomin, D. Frenkel, and V. N. Ryzhov, Phys. Rev. E 79, 051202 (2009).

[70] E. Lomba, N. G. Almarza, C. Martin, and C. McBride, J. Chem. Phys. 126, 244510 (2007).

[71] M. Girardi, M. Szortyka, and M. C. Barbosa, Phys. A (Amsterdam) 386, 692 (2007).

[72] D. Y. Fomin, N. V. Gribova, V. N. Ryzhov, S. M. Stishov, and D. Frenkel, J. Chem. Phys 129, 064512 (2008).

[73] N. M. Barraz, Jr., E. Salcedo, and M. C. Barbosa, J. Chem. Phys. 131, 094504 (2009).

[74] J. da Silva, E. Salcedo, A. B. Oliveira, and M. C. Barbosa, J. Chem. Phys. 133, 244506 (2010).

[75] L. B. Krott and M. C. Barbosa, J. Chem. Phys. 138, 084505 (2013)

[76] L. B. Krott and J. R. Bordin, J. Chem. Phys. 139, 154502 (2013).

[77] M. Chaplin, http://www.lsbu.ac.uk/water/anmlies.html.

[78] J. D. Weeks, D. Chandler, and H. C. Andersen, J. Chem. Phys. 54, 5237 (1971).

[79] P. Kumar, F. W. Starr, S. V. Buldyrev, and H. E. Stanley, Phys. Rev. E 75, 011202 (2007).

[80] W. G. Hoover, Phys. Rev. A 31, 1695 (1985).

[81] W. G. Hoover, Phys. Rev. A 34, 2499 (1986).

[82] M. Meyer and H. E. Stanley, J. Phys. Chem. B 103, 9728 (1999).

[83] P. Kumar, S. Han, and H. E. Stanley, J. Phys.: Condens. Matter 21, 504108 (2009)

[84] M. P. Allen and D. J. Tildesley, Computer Simulations of Liquids, 1st ed. (Clarendon, Oxford, 1987).

[85] P. Gallo, M. A. Ricci, and M. Rovere, J. Chem. Phys. 116, 342 (2002).

[86] A. Jabbarzadeh, P. Harrowell, and R. I. Tanner, Phys. Rev. Lett. 96, 206102 (2006)

[87] A. Jabbarzadeh, P. Harrowell, and R. I. Tanner, J. Phys. Chem. B 111, 11354 (2007)

[88] M. W. Maddox and K. E. Gubbins, J. Chem. Phys. 107, 9659 (1997).

[89] A. B. de Oliveira, E. B. Neves, C. Gavazzoni, J. Z. Paukowski, P. A. Netz, and M. C. Barbosa, J. Chem. Phys. 132, 164505 (2010). 